\title{
Twenty-six-year monitoring of water masers
}

\author{
G. M. Rudnitskij ${ }^{1}$, M. I. Pashchenko ${ }^{1}$, V. F. Esipov ${ }^{1}$, \\ V. A. Samodurov ${ }^{2}$, I. A. Subaev ${ }^{2}$, A. M. Tolmachev ${ }^{2}$ \\ and E. E. Lekht ${ }^{1,3}$ \\ ${ }^{1}$ Sternberg Astronomical Institute, Moscow State University, \\ 13 Universitetskij prospekt, Moscow, 119992 Russia \\ email: gmr@sai.msu.ru \\ ${ }^{2}$ Pushchino Radio Astronomy Observatory, Astrospace Center \\ of the Lebedev Institute of Physics, Russian Academy of Sciences, \\ Pushchino, Moscow Region, 142290 Russia \\ email: sam@prao.ru \\ ${ }^{3}$ Instituto Nacional de Astrofísica, Óptica y Electrónica, Luis Enrique Erro No. 1, \\ Apdo Postal 51 y 216, 72840 Tonantzintla, Puebla, México \\ email: lekht@inaoep.mx
}

\begin{abstract}
Since 1980 variability of a sample of $\mathrm{H}_{2} \mathrm{O}$ maser sources has been monitored on the 22 -metre radio telescope in Pushchino, Russia. The interval between successive observational sessions is 1-2 months. The sample includes 125 maser sources in star-forming regions (SFR) and late-type variable stars. Twenty-six-year time series of $\mathrm{H}_{2} \mathrm{O}$ line profiles have detected flares and velocity drift of spectral features. Very fast variations in the $\mathrm{H}_{2} \mathrm{O}$ maser flux $(\Delta t \lesssim 1$ hour) have been detected in several SFR sources, in particular, W33B. Variations of circumstellar $\mathrm{H}_{2} \mathrm{O}$ masers in late-type stars correlate with visual light curves with a time lag of $0.3-0.4 P(P$ is the star's period). Exceptionally strong $\mathrm{H}_{2} \mathrm{O}$ maser flares were recorded in SFR sources (Sgr B2 and others) and in the stars $\mathrm{W}$ Hya, $\mathrm{R}$ Cas and U Ori. Models for $\mathrm{H}_{2} \mathrm{O}$ maser variability are reviewed. For stellar masers shock-wave excitation of $\mathrm{H}_{2} \mathrm{O}$ line variability is discussed.
\end{abstract}

Keywords. molecular processes, radio lines: ISM, radio lines: stars, masers, surveys, stars: formation, stars: AGB and post-AGB, circumstellar matter, shock waves

Since 1980 our team has been monitoring cosmic maser sources radiating in the $6_{16}-5_{23}$ rotational line of the water vapour molecule at $\lambda=1.35 \mathrm{~cm}$ (Lekht et al. 1982; Berulis et al. 1983). The observations are carried out on the 22-metre radio telescope of the Pushchino Radio Astronomy Observatory. The sensitivity is about $10 \mathrm{Jy}$, the velocity resolution is $0.081 \mathrm{~km} / \mathrm{s}$. The interval between successive observational sessions is $1-$ 2 months.

The sample includes 125 maser sources of which 65 are in star-forming regions (Ori A, W75N, G43.8-0.1, NGC 2071 etc.) and the rest are in circumstellar envelopes of late-type variable stars (U Ori, RS Vir, RT Vir, U Her, VY CMa, VX Sgr etc.). For most sources $\mathrm{H}_{2} \mathrm{O}$ line profiles have been measured for more than 200 epochs between 1980 and 2006 .

For masers in star-forming regions the long time series have revealed some previously unknown regularities in the variations of the $\mathrm{H}_{2} \mathrm{O}$ line profiles (flares and velocity drift of individual spectral features). Figure 1 (left) presents examples of strong maser flares observed in the SFR source Sgr B2 (Ramírez Hernández et al. 2005). In some SFR sources (S255, NGC 7538) models of rotating proto-planetary discs and/or bipolar outflows are applicable (Lekht et al. 2004). Velocity drifts of maser features may reflect orbital motion of proto-planetary condensations. Dedicated observations allowed us to detect very fast variations in the maser flux of some sources (in particular, Sgr B2 and W33B) on a timescale shorter than 1 hour (Ramírez Hernández et al. 2005; Samodurov et al. 2007). 

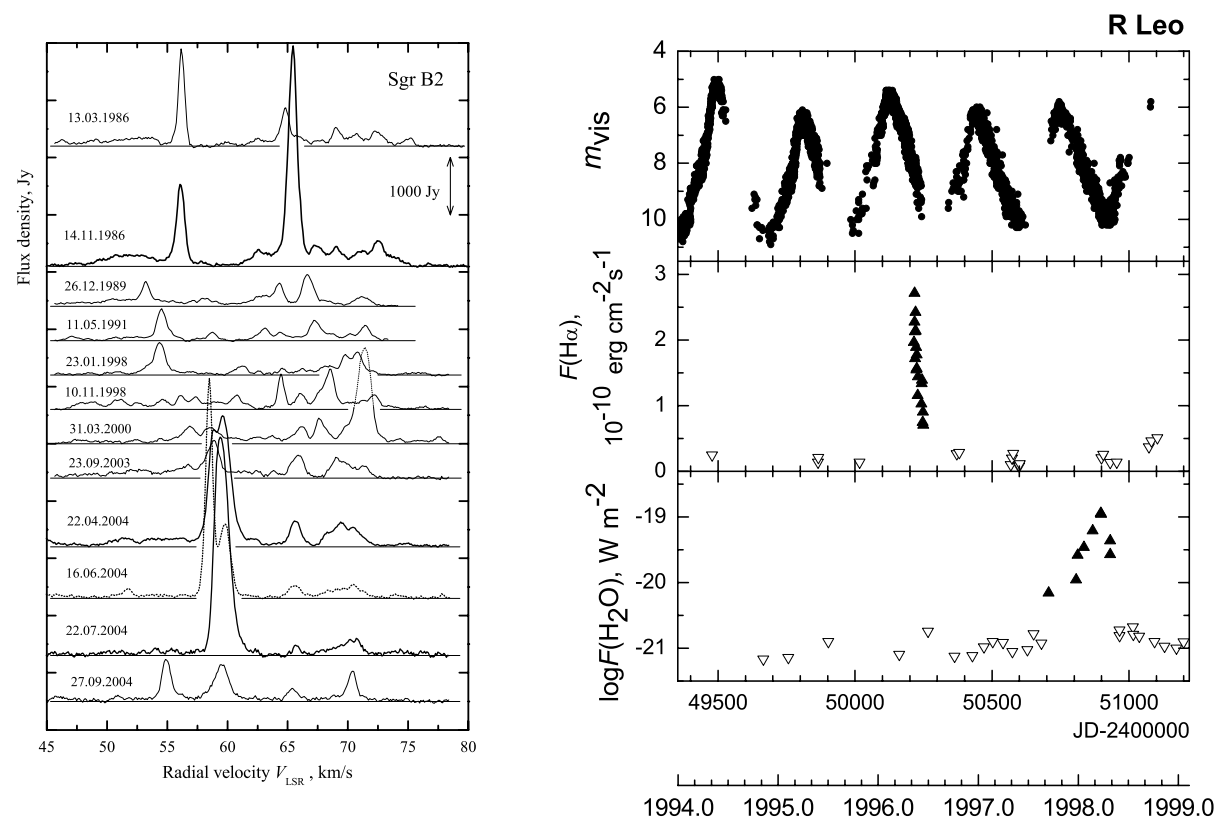

Figure 1. Flares of the $\mathrm{H}_{2} \mathrm{O}$ maser sources Sgr B2 and R Leo.

Variations of circumstellar $\mathrm{H}_{2} \mathrm{O}$ masers in late-type stars (U Ori, S CrB, U Her, RS Vir and others) correlate with the visual light curves with a time lag of $0.3-0.4 P$ ( $P$ is the star's period). Exceptionally strong $\mathrm{H}_{2} \mathrm{O}$ maser flares were noted in W Hya, R Cas and U Ori. Parallel optical spectroscopy of the stars has been carried out since 1994. Flares of the $\mathrm{H} \alpha$ line emission recorded in R Leo, U Aur, R Cas and R LMi were followed 1.52 years later by corresponding flares of the $\mathrm{H}_{2} \mathrm{O}$ masers. Figure 1 (right) shows an example of such a flare in the Mira-type star R Leo (Esipov et al. 1999). These phenomena are interpreted as a consequence of propagation of a shock wave driven by stellar pulsation. A strong shock propagating in the inner layers of a circumstellar envelope produces optical emission lines; further out, as the shock becomes weaker, it excites the $\mathrm{H}_{2} \mathrm{O}$ maser.

\section{Acknowledgements}

This work is supported by the Russian Foundation for Basic Research, project codes 0602-16806 and 07-02-08014. The RT-22 telescope is sponsored by the Ministry of Education and Science of the Russian Federation, facility registration number 01-10.

\section{References}

Berulis, I. I., Lekht, E. E., Pashchenko, M. I. \& Rudnitskij, G. M. 1983, Soviet Astron. 27, 179 Esipov, V. F., Pashchenko, M. I., Rudnitskij, G. M. \& Fomin, S. V. 1999, Astron. Lett. 25, 672 Lekht E. E., Pashchenko, M. I., Rudnitskij, G. M. \& Sorochenko, R. L. 1982, Soviet Astron. 26, 168

Lekht, E. E., Munitsyn, V. A. \& Tolmachev A. M. 2004, Astron. Rep. 48, 200

Ramírez Hernández, O., Lekht, E. E. \& Tolmachev, A. M. 2005, Astron. Rep. 49, 777

Samodurov, V. A., Rudnitskij, G. M., Tolmachev, A. M., Subaev, I. A., Lekht, E. E., Pashchenko, M. I. \& Logvinenko, S. V. 2007, Astron. Astrophys. Trans., in press 